\title{
Production of Dissimilar Metals Materials by the Method of Solid-State Joining
}

\author{
B. V. Borts, I. M. Korotkova, O. O. Lopata, V. I. Sytin, V. I. Tkachenko, I. O. Vorobyov \\ National Science Center "Kharkov Institute of Physics \& Technology", Kharkov, Ukraine \\ Email: borts@kipt.kharkov.ua
}

Received 23 April 2014; revised 23 May 2014; accepted 30 May 2014

Copyright (C) 2014 by authors and Scientific Research Publishing Inc.

This work is licensed under the Creative Commons Attribution International License (CC BY).

http://creativecommons.org/licenses/by/4.0/

cc) (i) Open Access

\begin{abstract}
Mechanisms taking place while hot rolling in vacuum of dissimilar materials joined in solid phase are described. It is shown that at joining of materials in solid phase redistribution of atoms from one material into another occurs on the interface of joining. On the base of calculation and experimental results it is concluded that the ultimate strength of the interface of joint is always higher than the ultimate strength of less durable material. Pair zirconium-stainless steel SS (Type AISI 321) and carbon steel (Type C22E) those are used in nuclear power are investigated.
\end{abstract}

Keywords

Vacuum Hot Roll Bonding, Solid Phase, Ultimate Strength, Interface

\section{Introduction}

Problems of service life and of safe operation of constructions, machines and units of nuclear power stations pose the tasks of strength and reliability increase for units and structural elements encourage development of new materials-dissimilar metals bonded. There is the necessity in materials with high strength and low density, weld ability with heterogeneous materials, wear resistance and electroconductivity, high-temperature resistance and corrosion resistance. None of existing materials [1], can't guarantee the needed complexity of properties. And only "laminar" multilayer allows to produce absolutely new material, properties of which differ from properties of its components. Production of safe and long-lived composition materials for unconnected joints of structural elements produced of materials with different properties is very topical task.

Together with fusion welding of materials, solid-state joining is important [2] method because it provides wide possibilities especially in specific conditions of the use. This method allows to produce [3] the necessary

How to cite this paper: Borts, B.V., Korotkova, I.M., Lopata, O.O., Sytin, V.I., Tkachenko, V.I. and Vorobyov, I.O. (2014) Production of Dissimilar Metals Materials by the Method of Solid-State Joining. Open Journal of Metal, 4, 40-47.

http://dx.doi.org/10.4236/ojmetal.2014.42005 
structures and properties of metals in the area of their joining: increased static strength, fatigue strength at high and low temperatures, corrosion resistance in corrosive media, corrosion-mechanical strength, serviceability under high heat loads and under different irradiations.

\section{Mechanisms That Proceed at Solid-State Joining of Heterogeneous}

During vacuum hot rolling activation of surfaces occurs at the expense of their shearing plastic deformation that promotes the fracture of oxide film on joined surfaces [4]. On pure (juvenile) surfaces activation [5] centers are produced [2] associated with the escape of dislocations on surface. If dislocations that provide the elastic deformation produce dipoles of wedge disclination and appear on metal surface into the area of physical contact then they are the center of disturbance. Each disclination may represent [6] effective way of fluxes of heterogeneous materials atoms directed towards each other. Mutual transport of atoms and energy transfer occurs by these channels that balances all the system. Further deformation of material causes the advance of metal atoms that have increased energy and displacing by produced channels of defected structure on long distance. In experiments atoms of metals were detected that were displaced into crystalline lattice of other material on depth up to $20 \mathrm{mkm}$. On interface of two metals the flux of heterogeneous atoms sets up directed in opposite directions. Due to this in the narrow range $(1-5 \mathrm{mkm})$ random rearrangement of two joining lattice occurs and the zone with mixed distribution of atoms of two materials forms on the interface of solid phase joint.

Investigation of joint of heterogeneous materials in solid phase by X-ray microanalysis had showed the presence of solid zones on boundaries of joints niobium-copper and niobium-zirconium. Even transition of one material into the other is observed on distance of several micrometers (Figure 1).

The performed investigations had showed that contacting surface layers of metal atoms may be considered as special two-dimensional solid solution. Dissolution of foreign atoms in crystalline lattice causes its distortion, variation of interatomic bonds and energy threshold for joining of metal atoms.

It is impossible to explain the joining of contacting metals only by mutual diffusion. It is refuted by the fact that joint may be obtained also between mutually insoluble metals (copper-lead, for instance).

Atom of metal belonging to one crystalline lattice may start the penetration in other under two conditions. The first condition-atoms of two metals must be approached to distances comparable with parameters of crystalline lattice. The second condition is the formation of declined dipoles and transfer of additional energy to atoms of metal, penetrating from one crystalline lattice to the other. After penetration of atoms the bonds of definite type form between them and atoms of the matrix of other bonded samples. After this the diffusion may proceed in usual manner.

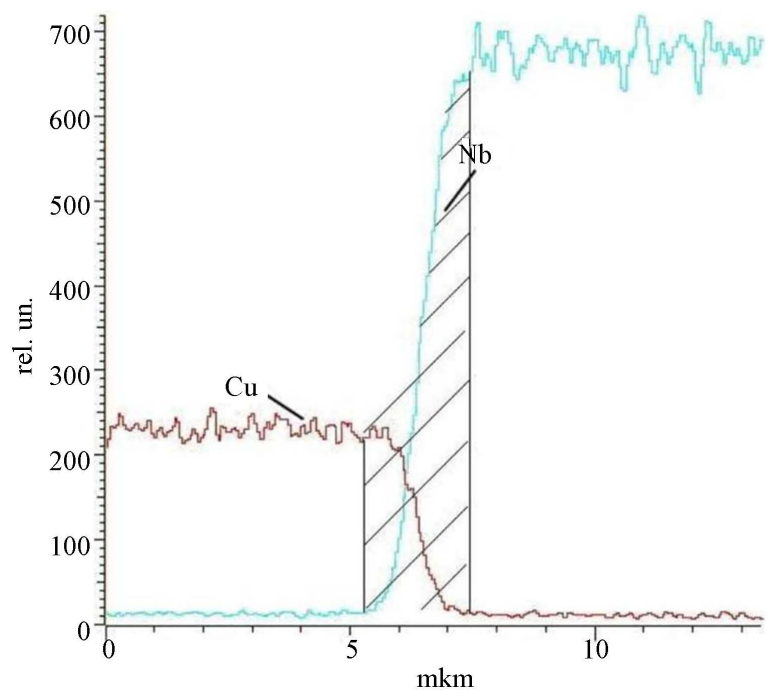

(a)

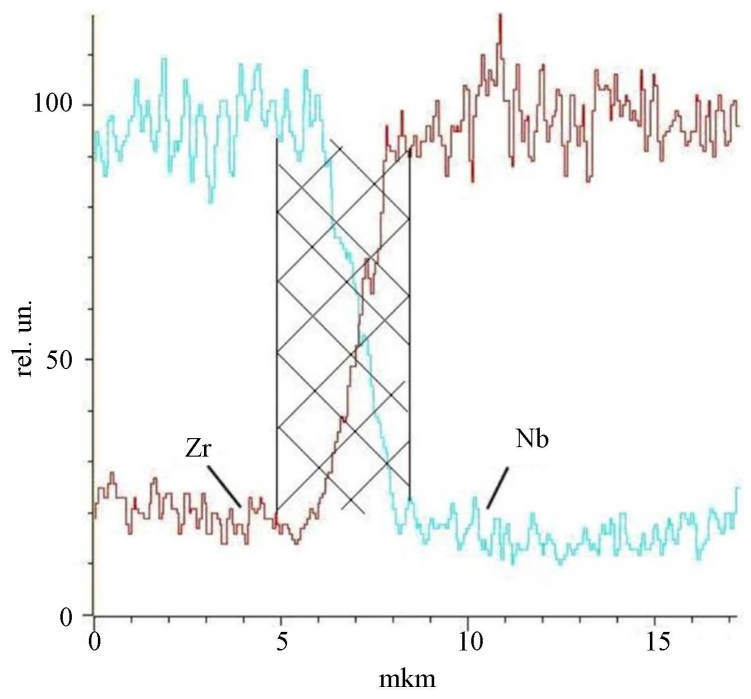

(b)

Figure 1. Spectra of micro X-ray spectrometric analysis near the interface of adjoint: (a) Niobium-copper; (b) Niobium-zirconium. 


\section{Determination of Relative Ultimate Strength of the Interface of Joint}

Theoretical model for ultimate strength of interface of heterogeneous materials joining in solid phase is described in details in [7]. In the presented paper we will consider only the final conclusions and statements. On joining of heterogeneous metals in solid phase the range of their strength variation will be in the limits [ $\sigma_{S p}{ }^{M}$ $\left.\sigma_{S 0}^{T}\right]$ where $\sigma_{S p}^{M}$-yield strength of less hard metal; $\sigma_{S 0}^{T}$-yield strength of more hard metal. Expression for relative ultimate strength of joining is.

$$
\left.\bar{\sigma}_{B}\left(t^{*}\right)\right|_{p}=\frac{k T}{E_{A}} \frac{\sigma_{S 0}^{T}}{\sigma_{S p}^{M}} \frac{\sigma_{S 0}^{T}+\sigma_{S p}^{M}}{\sigma_{S 0}^{T}-\sigma_{S p}^{M}} \ln \left(\frac{\sigma_{S 0}^{T}}{\sigma_{S p}^{M}}\right) \equiv \frac{k T}{E_{A}} Q_{p}\left(\frac{\sigma_{S 0}^{T}}{\sigma_{S p}^{M}}\right)
$$

where $k$-Boltzmann's constant; $E_{A}$-activation energy of plastic deformation of joined materials; $T$-temperature of rolling; $Q_{p}$-relative coefficient of strength of joined metals interface.

Introducing the coefficient of the strength range expansion $g=\sigma_{S 0}^{T} / \sigma_{S 0}^{M}$ we may represent expression (1) as a plot. Family of curves for dependencies of ultimate strength of interface of monometals joining on parameters of expansion of strength range $g$ was plotted.

Figure 2 shows the curve 1 that characterizes the strength of the interface of monometals joining. With the increase of limits of plasticity range of two different metals the strength characteristics of bimetals pair increases in comparison with monometallic one (see Figure 2, curves 2-4).

Such increase is observed until the range of bond strength will be determined by more hard metal. In this case the relative ultimate strength of sample bonding interface is determined by the ultimate strength of more hard material. Therefore the ultimate strength of interface of heterogeneous metals is considerably higher than the ultimate strength of soft metal and higher than the ultimate strength of multilayer material produced of soft monometals. The ultimate strength of heterogeneous metals interface is considerably higher than the ultimate strength of hard material but is lower the ultimate strength of multilayer material of hard monometals.

Figure 3 shows the tension diagram that shows that ultimate strength of joining interface is higher than the strength of less strength material. In this case the rupture of pair Stainless steel SS (Type AISI 321) and carbon steel (Type C22E) occurred by carbon steel under tension $430 \mathrm{MPa}$ that corresponds to the ultimate strength of carbon steel.

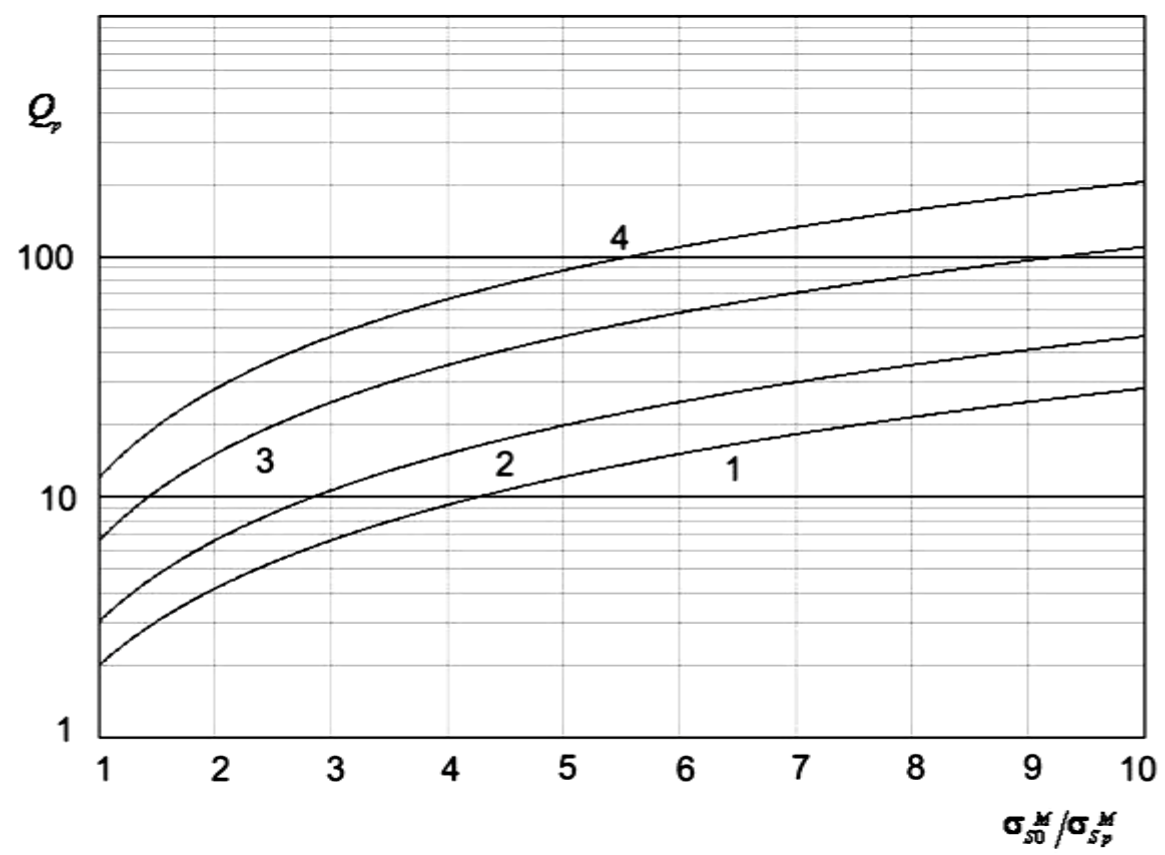

Figure 2. Dependence of relative strength coefficient of interface of joined metals on ratio of their yield strength $\sigma_{S 0}{ }^{M} / \sigma_{S p}{ }^{M}$ at different coefficients of yield strength range expansion: $g=1(1) ; g=1.5$ (2); $g=3$ (3); $g=5$ (4). 


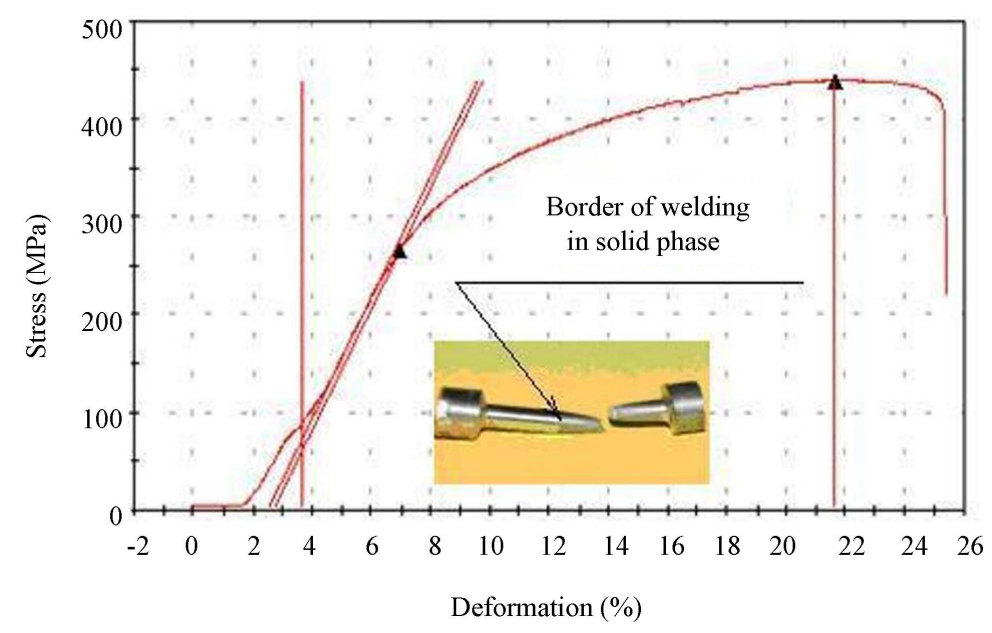

Figure 3. Tensile stress-deformation diagram of SS-C22E sample at $20^{\circ} \mathrm{C}$. The inset below shows the point of specimen fracture in steel C22E.

\section{Dissimilar Metals Solid-Phase Bonded for Use in Nuclear Engineering}

\subsection{Joint Zirconium-Stainless Steel}

To increase the service life and safe operation of assemblies and units of structures of reactor WWER-1000 high strength continuous joints of alloy zirconium-1\% niobium with stainless steel 12Cr18Ni10Ti, were produced by solid-state joining by high temperature rolling in vacuum.

Influence of working environment on the properties of multilayer material is investigated. Method of autoclave in liquid medium at temperatures and pressures simulating operation conditions was used. At autoclave testing two-times distilled water close by its composition to the coolant of first circuit of reactor WWER-1000 was used. Corrosion testing was carried out at temperature $350^{\circ} \mathrm{C}$ and under the pressure $16.5 \mathrm{MPa}$. Distilled water has considerably strong corrosion influence on materials due to the absence of inhibitors in this water.

Corrosion is the process of chemical or electrochemical interaction of metals with corrosion medium. Parameters of corrosion properties of solid phase-bonded materials components at autoclave were following: characteristic of polished metallographic specimen surface, namely, continuity, homogeneity of the film color, cracks and spallation; mechanical properties (strength and plasticity) of rupture specimens after different times of exposure in autoclave at operational regimes.

For investigation of corrosion micro metallographic specimens were polished and studied after testing during 50, 100, 500 and 1000 hours without preliminary etching structure of solid-phase bonded samples for monitoring the changes of surface of pair components.

Figure 4 and Figure 5 show the surfaces of the solid-phase bonding Zr1Nb-Ni-SS and Zr1Nb-Nb-SS before and after corrosion treatment.

For inseparable joint Zr1Nb-Ni-SS after rolling in initial state the transient zone of depth less $5 \mathrm{mkm}$ appears in nickel from side bordering on zirconium. After 50, 100, 500 and 1000 hours of testing the depth of transient zone remains invariable. Probably, the temperature of corrosion testing $\left(350^{\circ} \mathrm{C}\right)$ is insufficient for mutual diffusion of the solid-phase bonding elements on the interface of their mating.

With the increase of testing time some pitting corrosion occurs on the surface of nickel and also grain structure of nickel and zirconium manifests. Stainless steel remains invariable. Oxides of blue color are only observed around precipitates of remained $\alpha$-phase and carbides.

Study of mechanical properties of multilayer materials Zr1Nb-Nb-SS and Zr1Nb-Ni-SS were carried out at temperature $20^{\circ} \mathrm{C}$ in initial state and also after corrosion treatment in conditions of autoclave testing. Results of performed tests are shown on Figure 6.

As it is seen from presented data all compositions in initial state (after rolling) have rather high strength properties. The higher values of ultimate strength 445 - $465 \mathrm{MPa}$ are observed for composition ZrNb-Ni-SS. The fracture of specimen of multilayer material Zr1Nb-Ni-SS proceeds by interface zirconium-nickel. Multilayer material Zr1Nb-Nb-SS break down by interlayer of niobium. 


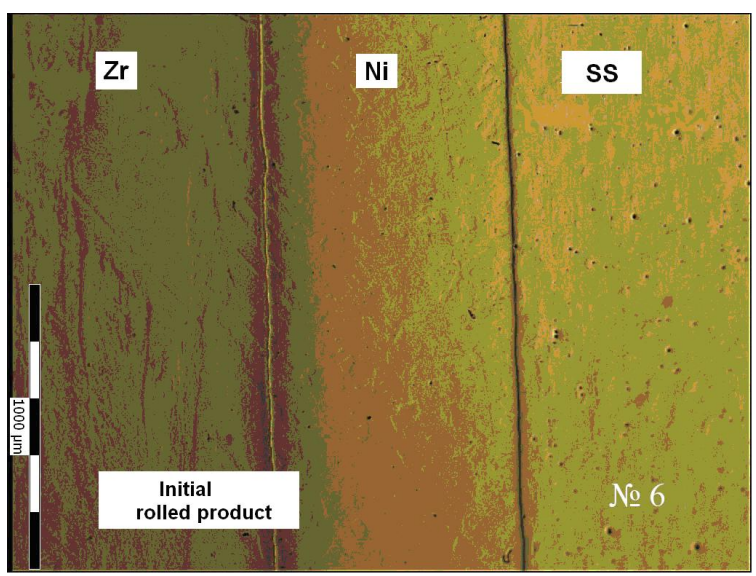

(a)

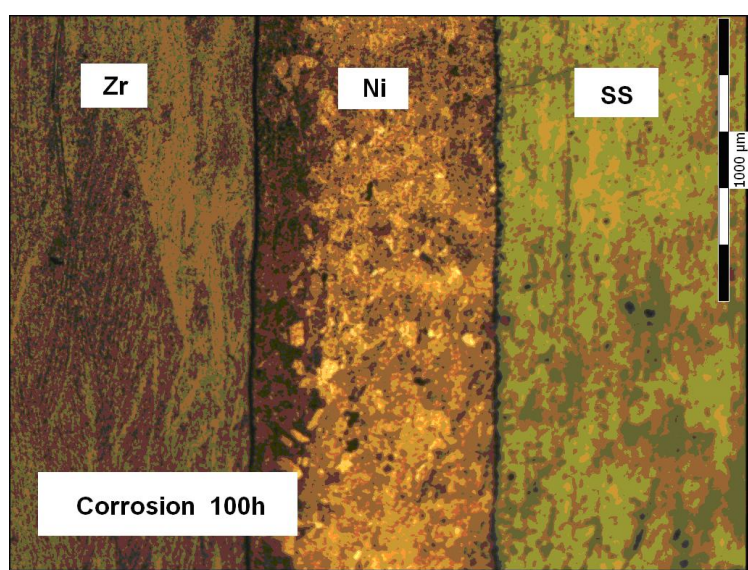

(b)

Figure 4. State of the surface of the solid-phase bonding Zr1Nb-Ni-SS: (a) In initial state; (b) After corrosion treatment during 100 hours.

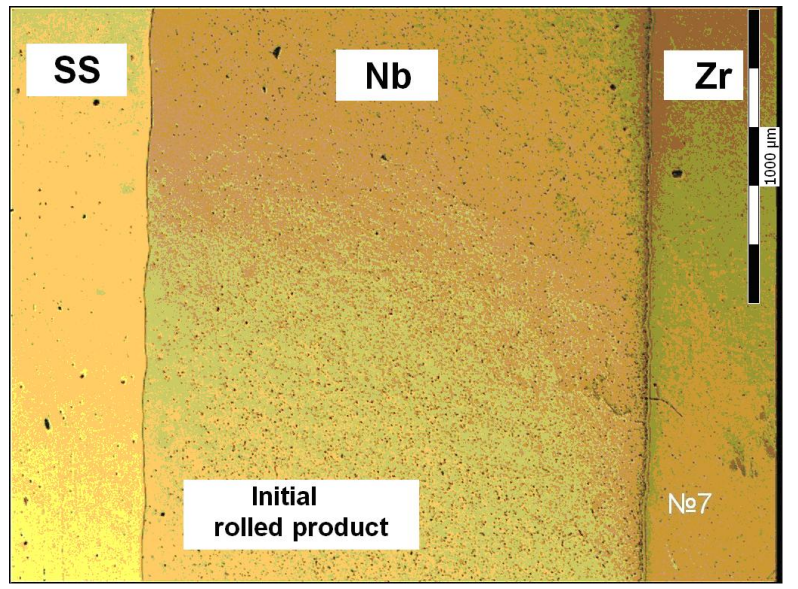

(a)

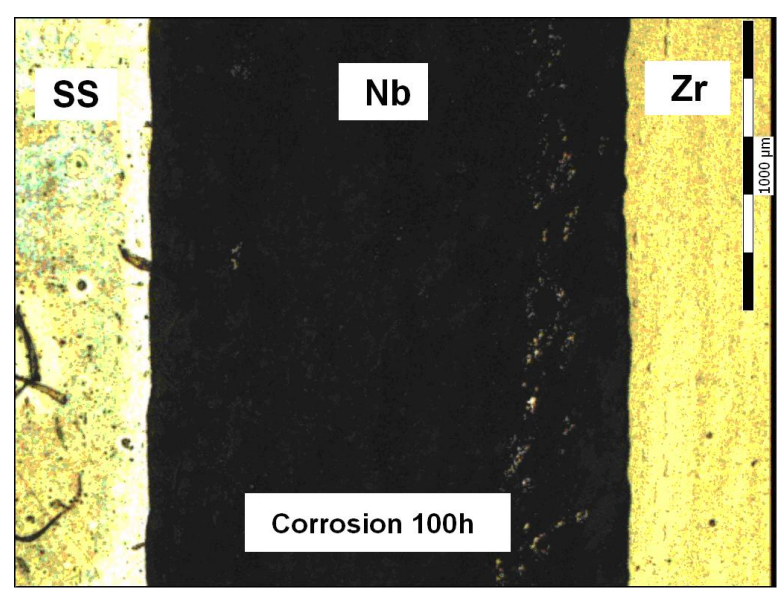

(b)

Figure 5. State of the surface of the solid-phase bonding Zr1Nb-Nb-SS: (a) In the initial state; (b) After corrosion treatment during 100 hours.

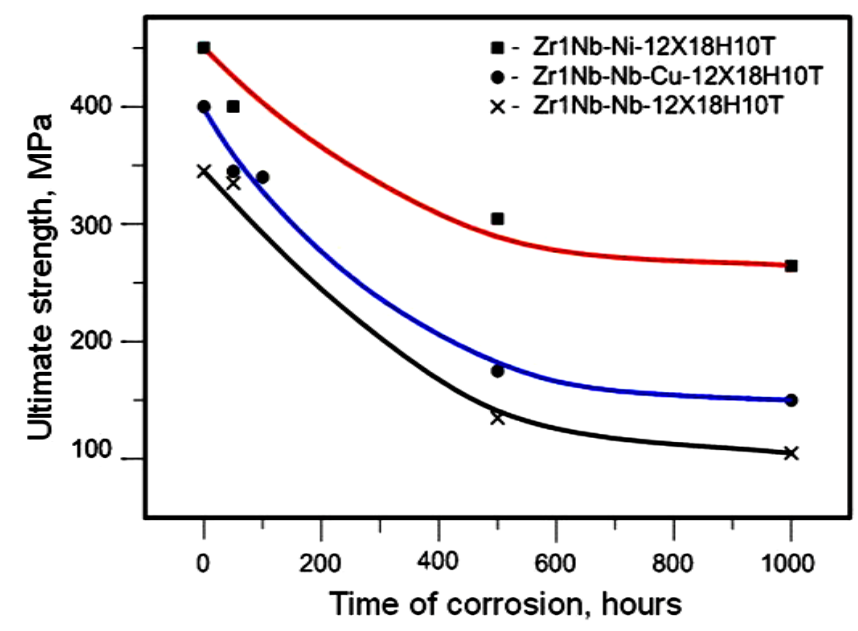

Figure 6. Dependence of ultimate strength of investigated composition on time of corrosion testing. 
After corrosion testing in water at operational temperature $350^{\circ} \mathrm{C}$ and pressure $16.5 \mathrm{MPa}$ considerable decrease of investigated compositions strength is observed. For Zr1Nb-Nb-12Cr18Ni10Ti the value of ultimate strength decreases from 340 - $350 \mathrm{MPa}$ in initial state to 100 - $110 \mathrm{MPa}$ after corrosion treatment during 1000 hours. Metallographic study had showed that during autoclave discontinuities as cracks on interface of interlayers with zirconium and steel were formed in the result of electrochemical corrosion. Separation into layers proceeds along specimen perimeter in the places of contact with corrosive medium. This causes the considerable decrease of joint strength and preliminary break down of the solid-phase bonding.

For Zr1Nb-Ni-SS decrease of strength is also observed after corrosion testing during 1000 hours $\left(\sigma_{b}=225\right.$ $280 \mathrm{MPa}$ ) that can be explained by the formation of transient zone on interface zirconium-nickel.

\subsection{Laminar Multilayer Material Stainless Steel SS (Type AISI 321) and Carbon Steel St20 (Type C22E)}

This material was produced in two manners: by solid-state joining of steel C22E (St20) and stainless steel Cr18Ni10Ti directly or through the interlayer of nickel. The temperature of rolling was $\left(980^{\circ} \mathrm{C} \pm 20^{\circ} \mathrm{C}\right)$ at relative reduction $(28 \% \pm 3 \%)$.

Temperature dependencies of mechanical characteristics of the solid-phase bonding steel 20-steinless steel (see Figure 7(a) and Figure 7(b)).

Testify the high strength of solid-state joining. Introduction of nickel as barrier interlayer doesn't influence considerably on values of ultimate strength and of yield strength of this material.

Metallographic analysis of the solid-phase bonding structure was performed with the use of metallographic complex (microscope GX-51 and analyzer of image IA-32). Figure 8 shows the microstructure of pair steel C22E-SS.

After vacuum rolling any additional phases were not detected on interface of joint steel C22E-stainless steel Cr18Ni10Ti. Microstructure of main components on interface differs from layers that are on depth. Near interface on distance of 20 - $30 \mathrm{mkm}$ from it new recrystallized grains have appeared with size 10 - $12 \mathrm{mkm}$ without signs of deformation in the grain body. In steel C22E decarburized zone (see Figure 8(a)) had appeared with columnar structure of ferrite with transverse dimensions $55-60 \mathrm{mkm}$ and not thick zone of finer equiaxial grains of ferrite with dimensions 35 - $40 \mathrm{mkm}$, passing to ferrite-perlite structure characteristic for low-carbonized steel (see Figure 8(a)).

After annealing ferritic zone in steel C22E increases in multilayer material rolled without interlayer. Dimensions of this zone depend on combination of temperatures and time of exposure. So, after annealing at $500^{\circ} \mathrm{C}$ during 10 hours substantial structural changes in comparison with the state after rolling are not observed neither in stainless steel nor in steel C22E (see Figure 8(b) and Figure 8(c)). High strength properties are preserved ( $\sigma_{b}$ $=400-440 \mathrm{MPa}$ at $20^{\circ} \mathrm{C}$ ). After lengthy exposure at temperatures $500^{\circ} \mathrm{C}$ and $600^{\circ} \mathrm{C}$ during 50 hours chemical

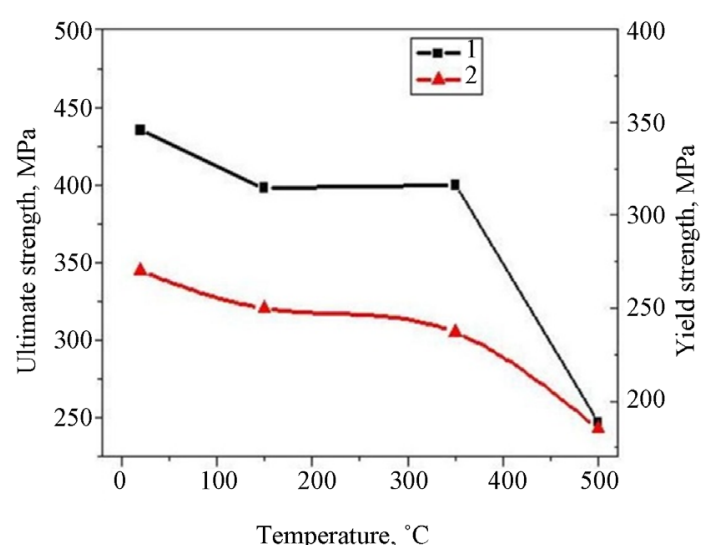

(a)

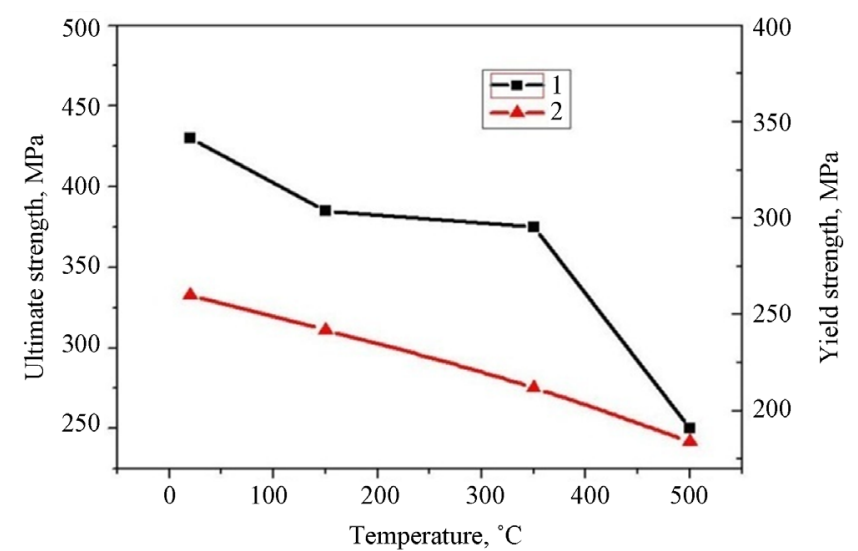

(b)

Figure 7. Temperature dependencies of mechanical characteristics of the solid-phase bonding steel C22E-SS (a) and steel C22E-Ni-SS (b); $1-\sigma_{b} ; 2-\sigma_{02}$. 


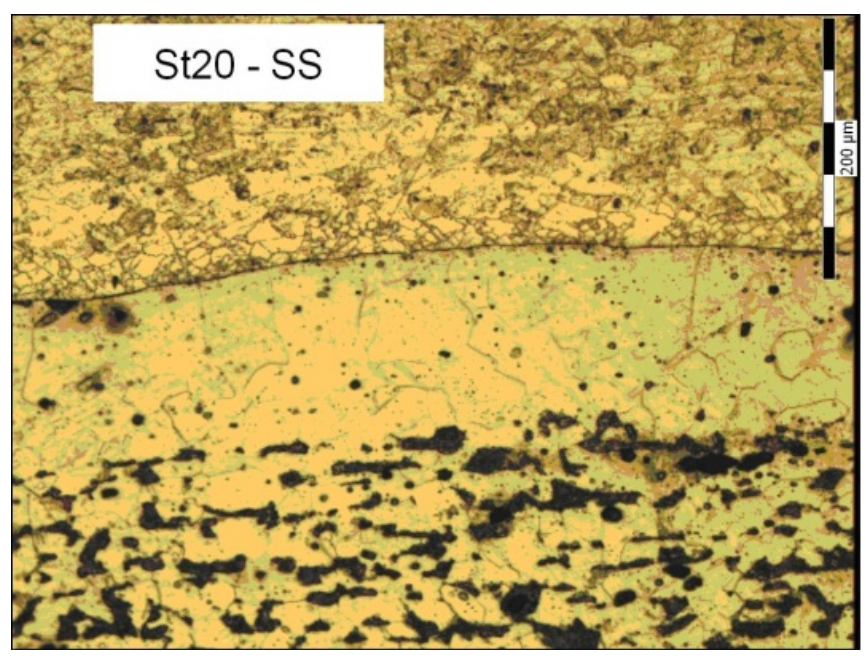

(a)

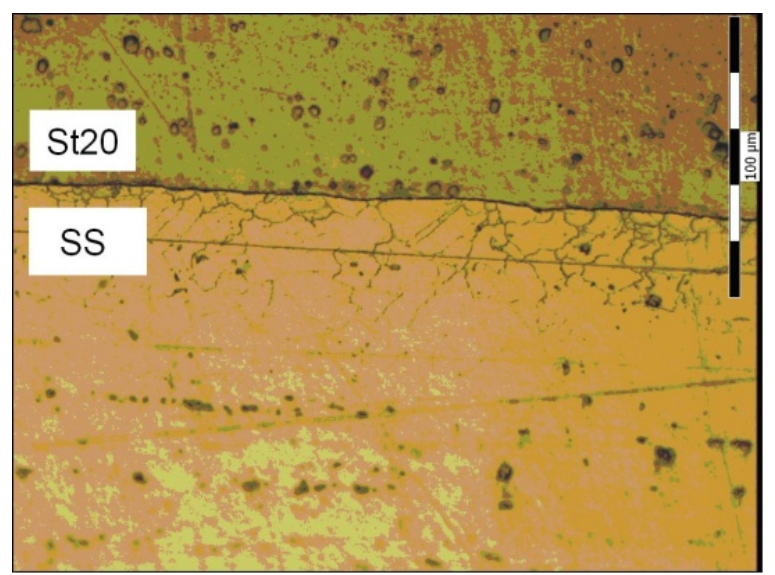

(b)

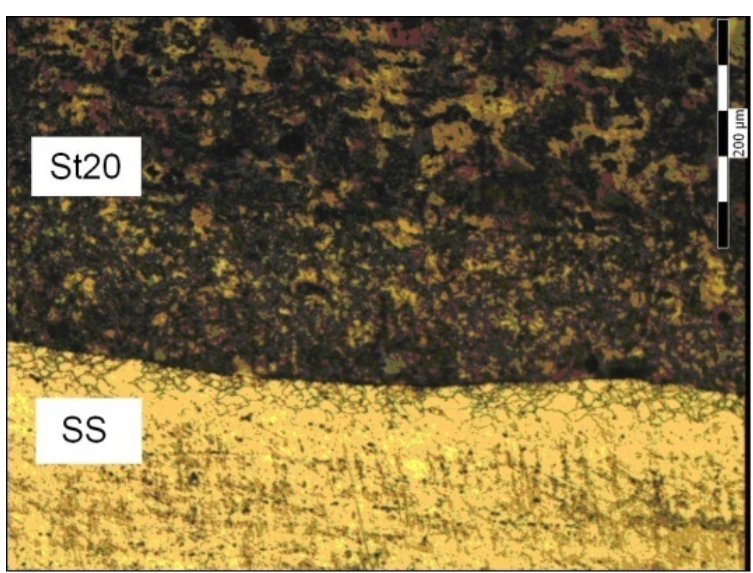

(c)

Figure 8. Microstructure of components of the solid-phase bonding steel C22E-SS ( $\times 200)$ : (a) On interface of layer joining; (b) Interface after annealing at $500^{\circ} \mathrm{C}$ during $10 \mathrm{~h}(\times 500)$; (c) Interface after annealing at $500^{\circ} \mathrm{C}$ during $10 \mathrm{~h}$ after durable etching $(\times 200)$.

and structural inhomogeneities are detected both in steel C22E and in stainless steel 12Cr18Ni10Ti. Zone of ferrite increases more than twice; from the side of stainless steel narrow light band appears with dimension of $\sim$ several micrometers, related with interdiffusion of multilayer material elements.

For the delay of carbon diffusion from steel C22E into the stainless steel the barrier interlayer of nickel was introduced into the solid-phase bonding. Microstructure of the solid-phase bonding steel C22E-Ni-SS is shown on Figure 9.

Analysis of specimen structure both after rolling and after thermal treatment had showed the absence of structural changes and mutual transport of elements on the interface of the solid-phase bonding steel C22ENi-SS.

\section{Conclusions}

1) The main mechanism responsible for material solid-state joining on rolling is the onset of rotational plasticity in the crystal and its fragmentation with the formation of joint disclinations.

2) It is possible to obtain solid-state joining by the method of hot rolling in vacuum. The ultimate strength of inhomogeneous metals interface exceeds essentially the ultimate strength of less strength metal.

3) This method of joining may be used for production of vacuum-proof and durable adapters, zirconium-steel, titanium-steel, low-carbon and stainless steel. 


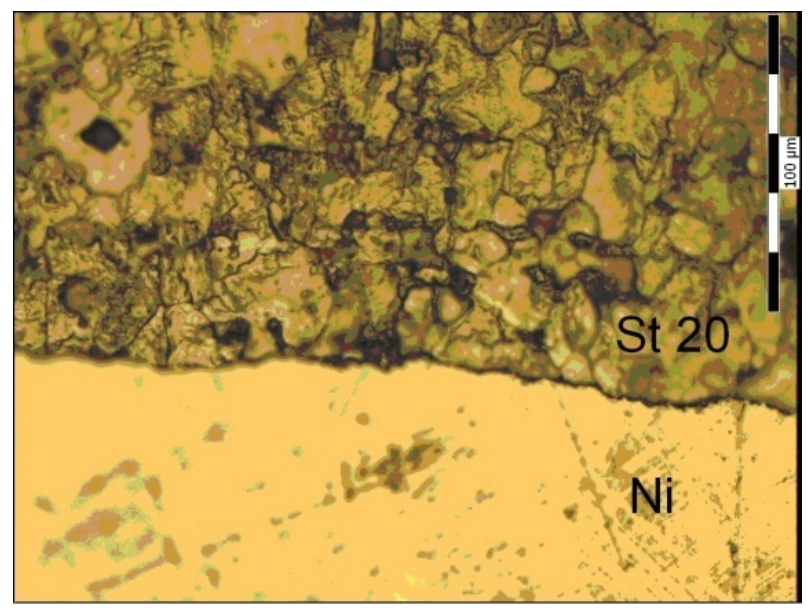

Figure 9. Microstructure of the solid-phase bonding C22E$\mathrm{Ni}-\mathrm{SS}$ on interface steel C22E -Ni after annealing at $500^{\circ} \mathrm{C}, 10$ h $(\times 200)$.

4) Zone of solid-state joining in multilayers the solid-phase bonding produced by the method of hot rolling in vacuum represents a new material with improved characteristics.

\section{References}

[1] Kundu. S. and Ghosh, M. (2005) Diffusion Bonding of Commercially Pure Titanium to 304 Stainless Steel Using Copper Interlayer. Materials Science and Engineering: A, 407, 154-160. http://dx.doi.org/10.1016/j.msea.2005.07.010

[2] Karakozov, E.S. (1976) Joining of Metals in Solid Phase. Metallurgiya, Moscow, 263 p.

[3] Thomas, K. (1994) Roll Welding. ASM Hand Book, 6, 312-314.

[4] An, J., Liu, Y.B., Lu, Y. and Sun, D. (2001) Hot Roll Bonding of Al-Pb Bearing of Alloy Strips and Hot Dip Aluminized Steel Sheet. Materials Characterization, 47, 291-297. http://dx.doi.org/10.1016/S1044-5803(01)00190-5

[5] Hwang, Y.M., Hso, H.H. and Lee, H.J. (1995) Analysis of Sandwich Sheet Rolling by Stream Function Method. International Journal of Mechanical Sciences, 37, 297. http://dx.doi.org/10.1016/0020-7403(95)93522-8

[6] Borts, B.V., Vanzha, A.F., Lopata, A.T., Neklyudov, I.M. and Shevchenko, S.V. (2005) Investigation of Processes of Welding of Multi Layers Structures of Crystals of Different Chemical Composition by Hot Rolling in Vacuum. Problems of Atomic Science and Technology, No. 5. Series: Physics of Radiation Damage and Radiation Material Science 88, 196-198.

[7] Borts, B.V. and Tkachenko, V.I. (2008) Phenomenological Description of Welding of Heterogeneous Metals in Solid Phase. Proceedings of XVIII International Conference on Physics of Radiation Phenomena and Radiation Material Science, Alushta, Crimea/Kharkov, 8-13 September 2008, 185-187. 